\title{
Responsabilidades en el hogar y salud de la mujer trabajadora
}

Gisela Blanco, M.Sc., ${ }^{(1)}$ Lya Feldman, M.Sc.(2)

\section{Blanco G, Feldman L. Responsabilidades en el hogar y salud de la mujer trabajadora. Salud Publica Mex 2000;42:217-225.}

\section{Resumen}

Objetivo. Analizar la relación existente entre la distribución de responsabilidades en el hogar y la salud física y mental percibida por un grupo de mujeres trabajadoras. Material y métodos Estudio transversal realizado en Caracas, Venezuela, entre septiembre de 1994 y octubre de 1995. Se encuestó a 260 profesoras universitarias so bre administración o planificación de las tareas en el hogar y realización de las mismas. Se calcularon medidas de tendencia central y se realizó análisis de varianza y regresión múltiple jerárquica. Resultados. En cuanto a la distribución de responsabilidades en el hogar, se observó que existe una mayor participación de las mujeres en la planificación y gerencia de las tareas y que reciben ayuda de su pareja en el mantenimiento de la casa.A quellas mujeres que informan una mayor carga en las tareas del hogar presentan ansiedad, depresión y baja autoestima. Conclusiones. Este hallazgo evidencia los efectos negativos que el trabajo del hogar puede tener sobre la salud mental en mujeres trabajadoras.

Palabras claves: salud de las mujeres; salud ocupacional; salud mental; trabajo de mujeres;Venezuela

\author{
Blanco G, Feldman L. \\ Home-making responsibilities \\ and health in working woman.
}

Salud Publica Mex 2000;42:217-225.

\begin{abstract}
A bstract
Objective. To analyze the relationship between the distribution of home-making responsibilities and perceived mental and physical health in working women. Material and methods A cross-sectional study was conducted between September 1994 and 0 ctuber 1995, in Caracas,Venezuela. A total of 260 univer sity teachers were surveyed on homemaking management and activities. Statistical analysis consisted of central tendency measures, analysis of variance and hierarchical multiple regression. Results C onsidering the distribution of home-making responsibilities, it was found that women participated more in home-making management and planning and that they received support from their partner in those tasks related to home-making. Those women who perceived higher overload in hometasks reported also higher levels of anxiety, depression and a lower self-esteem. Conclusions These results indicate the negative effects of household labor on mental health in working women.
\end{abstract}

Key words: women's health; occupational health; mental health; women, working;Venezuela
$\mathrm{L}$ a incorporación de la mujer al mercado de trabajo ha traído como consecuencia la necesidad de reformular los roles tradicionales de madre y esposa. ${ }^{1,2}$ Esta situación ha generado gran preocupación entre los investigadores de las ciencias sociales debido a las múltiples repercusiones no sólo en el funcionamiento familiar y laboral sino también en las condiciones de salud y bienestar de la mujer debido a que su carga familiar no ha variado en términos de responsabilidades, manejo, administración y ejecución de las tareas

(1) Escuela de Salud Pública, Facultad de Medicina, Universidad C entral, C aracas,Venezuela.

(2) Departamento de Ciencia y Tecnología del Comportamiento, Universidad Simón Bolívar, Caracas,Venezuela.

Fecha de recibido: 27 de agosto de 1999 - Fecha de aprobado: 18 de abril de 2000

Solicitud de sobretiros: Lya Feldman. Departamento de Ciencia y Tecnología del Comportamiento, Universidad Simón Bolívar. A partado Postal 89000, 1080, Caracas, Venezuela.

Correo electrónico: Ifeldman@ usb.ve; y giblanco@ eldish.net 
del hogar. Es por ello que el estudio de las características de la distribución de responsabilidades en el hogar ha adquirido relevancia en los últimos años debido al impacto que esta variable tiene en la dinámica familiar. $^{3-5}$

En la mayoría de los casos, la mujer que trabaja fuera del hogar lleva a cabo una serie de tareas dentro de la casa que incluye el cuidado de los hijos lo que implica que su jornada laboral se duplique. ${ }^{6,7}$

Las responsabilidades en el hogar han sido definidas básicamente de dos maneras. Algunos autores ${ }^{4}$ consideran el trabajo en el hogar como la participación en las tareas propias de la casa: preparar las comidas, el aseo o limpieza, las reparaciones menores de la casa, entre otras. Otros autores incluyen, además, el cuidado de los hijos como parte de las responsabilidades en el hogar. ${ }^{8}$

El hecho de englobar el cuidado de los hijos como una responsabilidad más de la casa y no como una función puramente femenina ha permitido involucrar a las parejas o esposos de las mujeres trabajadoras en estas actividades. Wiersma y Van Den Berg ${ }^{3}$ notifican una asociación mínima entre el número de horas que la mujer trabaja fuera de la casa y la cantidad de tiempo que dedica su esposo a las responsabilidades del hogar. Sin embargo, cuando se incluyen las horas dedicadas al cuidado de los hijos se aprecia un incremento considerable en la participación masculina. Rosenfield ${ }^{9}$ afirma que más de $50 \%$ de las tareas del hogar involucran el cuidado de los hijos. Es por ello que la tendencia actual es incluir dentro del conjunto de las actividades en el hogar las relacionadas con el cuidado de los hijos. ${ }^{5}$

En este estudio se utilizará la definición empleada por Baruch y Barnett ${ }^{8}$ que incluye el cuidado de los hijos dentro de las responsabilidades del hogar; se toma en consideración que se evaluará no sólo la participación en las actividades o tareas del hogar por parte de cada miembro de la pareja, sino la diferenciación sugerida por Mederer ${ }^{5}$ con relación a la organización o planificación de las tareas y la ejecución de las mismas en forma separada.

Las mujeres que trabajan fuera del hogar perciben beneficios tanto materiales como psicológicos que redundan en su desarrollo profesional y personal. Pero cuando a la mujer se le agregan las responsabilidades familiares, éstas pueden representar altas demandas que a su vez pueden influir en sus condiciones de salud y en el nivel de bienestar general, incluyendo la satisfacción marital. ${ }^{10}$ Estas tareas del hogar se hacen progresivamente rutinarias, aburridas y representan una gran carga para la mujer, a esto contribuye que se realicen generalmente en aislamiento social. A esta situación se suma el conflicto de intereses entre el trabajo exterior y el de la casa lo que genera agotamiento y tensión, especialmente si la mujer no percibe apoyo de su pareja en cuanto a la participación de éste en el hogar. Yogev y Brett ${ }^{11}$ encontraron que las mujeres trabajadoras tenían altos niveles de satisfacción marital cuando sus esposos participaban en las actividades del hogar. Ross, citado por Baruch y Barnett, ${ }^{8}$ afirma que la participación de los esposos en las tareas del hogar es predictor de ausencia de depresión y mejor percepción de salud en sus esposas. Por su parte, Piña y Bengtson ${ }^{7}$ señalan que las esposas que perciben una distribución equitativa de las responsabilidades del hogar respecto a sus esposos refieren sentirse satisfechas con sus parejas y perciben un estado de bienestar general.

Muchas mujeres que comparten su tiempo de trabajo con sus responsabilidades en el hogar solucionan la tensión generada por ambos roles trabajando turnos parciales. Sin embargo, tomar esta opción les impide lograr un mayor desarrollo profesional. Estas consecuencias se han visto mediatizadas por una serie de condiciones asociadas como son la participación por parte del esposo en las tareas del hogar, el nivel de desarrollo profesional a que aspire la mujer, la edad y el número de hijos; condiciones éstas que pueden servir como factores de riesgo tanto para su salud en general como para valorar su nivel de satisfacción individual y con su pareja., 71,12

El presente estudio se planteó como objetivo fundamental estudiar la relación entre la distribución de responsabilidades en el hogar y la salud mental y física percibida por mujeres profesionales universitarias, considerando la relación estrecha de esta variable con los índices de bienestar general. Se esperaría que aquellas mujeres que tienen una sobrecarga en las tareas del hogar, informen un peor estado de salud tanto físico como mental.

\section{Material y métodos}

Este estudio se realizó entre septiembre de 1994 y octubre de 1995. Fueron 125 docentes participantes, aunque la muestra inicial fue de 260 , pertenecientes a tres centros de educación superior de la zona metropolitana de Caracas, quienes desempeñan roles múltiples (rol laboral: dedicadas a actividades docentes o de investigación de tiempo completo o integral; rol de pareja: casada o unida, y rol de madre).

Se utilizaron cuatro cuestionarios de autoinforme. El instrumento para medir la distribución de responsabilidades en el hogar consta de una lista de actividades a realizar en el hogar por cada uno de los 
integrantes y más específicamente a la frecuencia con la que ejecutan las actividades la mujer, su pareja u otras personas. Se divide en dos partes: a) administración o planificación de las tareas, el cual consta de siete ítemes, y b) realización de las tareas, que contiene 23 ítemes. Este cuestionario fue diseñado por Feldman, Chacón, Blanco y Carrasquel* con base en el propuesto por Mederer,; se evaluó consistencia interna para el manejo y planificación de las tareas y para la realización de las mismas por medio del coeficiente de consistencia interna $\alpha$ de Cronbach.

Con el objeto de determinar la validez de constructo de este instrumento se realizó un análisis factorial utilizando el método de componente principal con matriz de rotación Equamax. Para la dimensión correspondiente a la planificación de las actividades del hogar se obtuvieron dos factores (planificación-decisiones y alimentación). Con relación a la realización de las tareas fueron cuatro los factores resultantes (tareas relacionadas con el hogar, cuidado de los niños, otras tareas y mantenimiento del hogar).

El cuestionario de datos sociodemográficos recabó información sobre estado civil, nivel de instrucción, horas semanales de trabajo, fuentes de ingreso, condiciones de la vivienda, datos de la pareja, número de hijos, hijos en edad escolar, edad, nivel educativo y ocupación de ambos padres de la mujer, así como aspectos del cuidado y manutención de los hijos.

La salud física se evaluó utilizando información referente a la autopercepción de la persona así como de un indicador de morbilidad. La autopercepción de salud fue calificada (sobre la base de una escala de cinco puntos) por la participante como: excelente, muy buena, buena, deteriorada y muy deteriorada; así como la comparación de esta percepción relacionada con otras personas de su misma edad en la actualidad y cinco años atrás, utilizando la misma escala. En los indicadores de morbilidad se tomó en cuenta información acerca de si había consultado o no a un médico o algún profesional de la salud durante los últimos seis meses y cuántas veces; si había estado hospitalizada en los últimos cinco años y en los últimos seis meses, y cuántos días había faltado al trabajo y permanecido en cama por motivos de salud en los últimos seis meses.

Por otra parte, para evaluar los hábitos de salud relacionados con factores de riesgo y protectores de ciertas enfermedades se interrogó sobre: hábito tabá-

\footnotetext{
* Feldman L, Chacón G, Blanco G, Carrasquel Y. Proyecto de investigación mujer-trabajo y salud. Decanato de Investigaciones. Caracas: Universidad Simón Bolívar, 1995. Publicación interna.
}

quico, cantidad de cigarrillos diarios, práctica de algún deporte y su frecuencia, consumo de alcohol y cantidad, y horas diarias de sueño.

Para evaluar la salud mental se tomaron en cuenta tres aspectos: ansiedad, depresión y autoestima. Para medir la ansiedad se utilizó el cuestionario de Spielberger y colaboradores, * utilizando únicamente el factor de ansiedad de estado, mismo que consta de 20 afirmaciones con base en una escala de cuatro puntos, en el cual $0=$ no en lo absoluto, $1=$ un poco, $2=$ bastante y $3=$ mucho. Este cuestionario ha sido ampliamente utilizado en muestras de habla hispana y se ha obtenido una confiabilidad de 0.93 utilizando el coeficiente de consistencia interna $\alpha$ de Cronbach. ${ }^{\ddagger}$

En cuanto a la depresión se utilizó un cuestionario que consta de ocho ítemes basado en el diseñado por Derogatis y colaboradores ${ }^{13}$ encontrándose valores apropiados de consistencia interna de $0.72 . \ddagger$ Para el indicador de autoestima se utilizó el instrumento diseñado por Johnson ${ }^{14}$ el que está conformado por ocho ítemes relacionados con la percepción afectiva de sí mismo y la percepción que tienen los otros, la confiabilidad obtenida fue de 0.85 .

Se incluyó un listado de reporte de síntomas basado en el cuestionario de excitabilidad individual, adaptado por Canino, Groeger y Robles ${ }^{15}$ y modificado por Feldman, $\ddagger$ el que quedó reducido sobre la base de un análisis factorial a 13 ítemes, con una escala de $0=$ ausencia y $1=$ presencia del síntoma, con lo que se obtuvo un índice de consistencia interna de 0.74 .

La ubicación del personal docente de las diferentes instituciones se realizó mediante los listados de nómina, facilitados por las diferentes direcciones de recursos humanos, los que contenían el nombre del docente, cargo que desempeña y dependencia a la que pertenece. Luego de seleccionar al personal docente femenino que permanecía en la universidad toda la jornada laboral, específicamente profesoras con dedicación exclusiva e integral (seis a ocho horas diarias), se procedió a codificar los cuestionarios otorgándoles códigos diferentes dependiendo de la institución a la cual pertenecían.

Para la entrega de los cuestionarios se realizaron reuniones con los jefes de cada escuela, departamento

\footnotetext{
* Spielberger ChD, González-Reigosa F, Martínez-Urrutia A, Natalicio LFS, Natalicio, DS. Development of the Spanish Edition of the State-Trait Anxiety Inventory. Miami: Florida State University, 1971. Manuscrito.

‡ Feldman L, Chacón G, Blanco G, Carrasquel Y. Proyecto de investigación mujer-trabajo y salud. Decanato de Investigaciones. Caracas: Universidad Simón Bolívar, 1995. Publicación interna.
} 
o unidad; aquellos a quienes no se pudo localizar personalmente se les hizo llegar una carta con la finalidad de informarles de las características y objetivos del proyecto y los cuestionarios en un sobre cerrado. A cada participante se le asignó un código con el fin de garantizar la confidencialidad. Asimismo, en la comunicación se les dieron instrucciones con relación al proceso de devolución de los cuestionarios.

El procesamiento estadístico se realizó por medio del Statistical Package for Social Science (SPSS, por sus siglas en inglés), versión 6.0. El análisis descriptivo incluyó medidas de tendencia central (media y desviación estándar) y distribución de las variables por medio de los porcentajes. Con el fin de comparar la distribución de responsabilidades en el hogar de acuerdo con el tipo de rol (sin pareja con hijos, con pareja sin hijos y con pareja e hijos) se utilizó un análisis de varianza (Anova) con el método de comparaciones múltiples post hoc, utilizando el LSD* modificado y la prueba de Bonferroni. Para evaluar la relación entre la distribución de responsabilidades en el hogar y la salud se realizó análisis de regresión lineal. Estas medidas se elaboraron al controlar los efectos de: edad de las mujeres, hijos en edad escolar, horas semanales de trabajo y tipo de cargo. En un primer paso se evaluó la relación entre estas últimas variables (a controlar) y la variable salud (a predecir). En un segundo paso se incluyeron los factores de la variable distribución de responsabilidades en el hogar, con lo que se evaluó si la entrada de ésta era significativa incluso después de controlar el efecto de las variables sociodemográficas sobre las variables de salud. Para cada caso se informó $\chi^{2}$ y para cada variable, el coeficiente de regresión estandarizado (beta). En tanto que para el modelo global se señaló la significancia dada por medio de la prueba $\mathrm{F}$ que se realiza sobre la ecuación de regresión.

*LSD. Procedimiento que se utiliza para afinar y alcanzar mayor precisión al análisis de varianza (Anova).

\section{Resultados}

El porcentaje de respuesta fue de $48 \%$. Como se observa en el cuadro I puede afirmarse que la consistencia interna del cuestionario sobre distribución de las responsabilidades en el hogar es adecuada, con un coeficiente de 0.72 para el manejo y planificación de tareas, y de 0.87 para la realización de las mismas. Si bien podría pensarse que el índice de manejo o planificación de las actividades es relativamente bajo, esto se debe a que el cuestionario de responsabilidades en el hogar, específicamente el que corresponde al manejo o planificación de actividades, tiene un menor número de ítemes (siete en total). Para un menor número de ítemes, los valores $\alpha$ de Cronbach aceptables son también menores, por ejemplo, para un cuestionario de siete ítemes o menos un $\alpha>0.60$ puede ser muy adecuado. Asimismo, el análisis factorial muestra que explica 63.8 y $57.7 \%$ de la varianza, respectivamente.

En cuanto a datos sociodemográficos se encontró la siguiente combinación de roles múltiples: 33 mujeres $(26.8 \%)$ trabajaban y tenían pareja; 23 trabajaban y eran madres $(18.7 \%)$, y 67 mujeres ejercían los roles trabajo-pareja-madre (54.5\%). Las edades de las participantes oscilaron entre 25 y 72 años, con un promedio de cuarenta años $(40 \pm 8.86)$.

Todas las participantes tienen estudios universitarios completos, y 31.7\% ocupaba cargos de jefatura o gerenciales. El $75.6 \%$ de las mujeres estaban casadas; $12.2 \%$ reportó ser divorciada; $5.7 \%$, unida o conviviendo con una pareja sin estar casada; $2.8 \%$, separada, y $3.7 \%$, viuda. Las edades de las parejas oscilaron entre 25 y 76 años, con un promedio de 42 años, $(42.9 \pm 10.18)$. Con relación al nivel educativo de las parejas se encontró que la mayoría (88.3\%) tenía estudios universitarios completos; $5.1 \%$ informó tener estudios universitarios incompletos; $3.3 \%$, estudios secundarios completos, y $3.3 \%$, estudios secundarios incompletos. Con relación al número de hijos se encontró que $36.6 \%$ de las participantes tienen un hijo;

\section{Cuadro I
INDICE DE CONFIABILIDAD Y ESTADISTICOS DESCRIPTIVOS DEL CUESTIONARIO SOBRE DISTRIBUCIÓN de las responsabilidades en el hogar. Caracas, Venezuela, 1994-1995}

$\begin{array}{lccccc}\text { Variable } & \text { Alfa de Cronbach } & \text { Promedio } & \text { Desviación estándar } & \text { Rango esperado } & \text { Rango posible } \\ \begin{array}{l}\text { Responsabilidades en el hogar } \\ \quad \text { Organización }\end{array} & 0.72 & 33.85 & 4.19 & 16-42 & 7-42 \\ \begin{array}{l}\text { Responsabilidades en el hogar } \\ \text { Ejecución }\end{array} & 0.87 & 88.15 & 11.96 & 62-128 & 22-132 \\ n=125 & & & & \end{array}$


$27.6 \%$, dos hijos; $22.8 \%$, no tiene hijos y un menor porcentaje $(13 \%)$, tres hijos. Un poco más del tercio de las mujeres (33.3\%) tiene hijos en edad escolar.

En relación con la salud física, la mayoría de las mujeres participantes informaron percibir su salud como excelente $(10.5 \%)$, muy buena $(43.1 \%)$, buena $(42.3 \%)$, y deteriorada (4.1\%). Ninguna notificó su salud como muy deteriorada.

Al comparar su salud con las otras personas de la misma edad, la mayoría de las mujeres consideraron que era igual (61\%), y sólo un pequeño número de ellas la consideraron peor $(2.4 \%)$. Comparando su salud actual con la de cinco años atrás, un poco más de la mitad de las participantes la consideraron igual (54.9\%), 32\% la percibió como peor y $13.1 \%$ comunicó estar en mejores condiciones de salud.

Con relación a la asistencia a consultas médicas en los últimos seis meses, $34.1 \%$ de las mujeres asistieron una vez; $33.3 \%$ no asistieron a ninguna consulta; $18.9 \%$, dos veces; $8.9 \%$, tres veces, y $4.8 \%$, cuatro veces. En cuanto a la asistencia a consultas psicológicas o psiquiátricas, $94.3 \%$ notificó no haber asistido a tales consultas mientras que $5.7 \%$ asistió una sola vez.

La mayor parte de las participantes (91.1\%) no habían sido hospitalizadas en los últimos seis meses, mientras que $8.9 \%$ refirió haber estado hospitalizada al menos una vez.

El número de faltas al trabajo debido a problemas de salud fue relativamente bajo, $61.7 \%$ no faltó ningún día al trabajo por problemas de salud; $11.7 \%$ faltó una vez; $10.8 \%$, dos veces; $10.8 \%$, tres veces, y $5 \%$, faltó al trabajo cinco veces. El $61.2 \%$ de las participantes informó no haber permanecido en reposo en la cama durante los últimos seis meses; $17.4 \%$ refirió haber permanecido un día en cama; $12.7 \%$, dos días; $8,6 \%$ reportó tres días y un porcentaje similar cinco días.

En cuanto a la presencia de enfermedades en las participantes fueron muy bajas: asma, $10 \%$; bronquitis, $8.3 \%$; úlcera, $5 \%$, e hipertensión, $3.4 \%$, entre las más importantes.
En relación con los hábitos de salud, del total de las participantes, $84.6 \%$ eran no fumadoras al momento de la evaluación; sin embargo, $47.5 \%$ había fumado en el pasado. El $90 \%$ de las mujeres fumadoras aspiraban al fumar y $10.5 \%$ fumaba de uno a seis cigarrillos diarios, $1.6 \%$ fumaba veinte cigarrillos o más al día. El $51.7 \%$ refirió estar en lugares donde otros fuman, considerándose fumadoras pasivas.

Con relación al ejercicio físico o la práctica de deportes poco más de la mitad de las mujeres participantes practica algún tipo de deporte (54.1\%), de los cuales, $72.1 \%$ eran ejercicios o deportes aeróbicos, $9.8 \%$ realizaba deportes o ejercicios anaeróbicos y $8.5 \%$ de ambos tipos.

Del total de las participantes, $61 \%$ notificó no consumir bebidas alcohólicas. El 36.6\% refirió consumir de una a cinco copas semanales de alcohol y $2.4 \%$, de seis a 10 copas por semana.

En relación con las horas de sueño diarias, la mayoría $(63.4 \%)$ duerme de siete a nueve horas diarias, y $35.8 \%$ duerme de cuatro a seis horas diariamente. Más de la mitad de las participantes (56.1\%) informaron no tener problemas de sueño.

En cuanto al estado de salud mental y reporte de síntomas, como puede observarse en el cuadro II, las mujeres sin pareja, con hijos, presentan mayores niveles de ansiedad y depresión en comparación con las mujeres con pareja, sin hijos, y las mujeres que desempeñan los tres roles. No se observaron diferencias significativas entre los grupos para las variables autoestima y reporte de síntomas.

En cuanto a la distribución de las responsabilidades en el hogar según el tipo de roles, se encontró en las comparaciones post hoc que las mujeres sin pareja, con hijos, tienen mayores responsabilidades tanto en la planificación como en la realización de las actividades de la casa, en comparación con las mujeres que tienen pareja con o sin hijos (cuadro III).

Los resultados de las regresiones para relacionar las variables sociodemográficas con los indicadores de

\section{Cuadro II \\ INDICADORES DE SALUD MENTAL Y REPORTE DE SÍNTOMAS SEGÚN TIPO DE ROLES. Caracas, Venezuela, 1994-1995}

\begin{tabular}{|c|c|c|c|c|c|c|c|}
\hline \multirow[b]{2}{*}{ Salud mental y síntoma } & \multicolumn{2}{|c|}{ Sin pareja, con hijos $n=23$} & \multicolumn{2}{|c|}{ Con pareja, sin hijos $n=33$} & \multicolumn{2}{|c|}{ Con pareja, con hijos $n=67$} & \multirow[b]{2}{*}{ Anova } \\
\hline & Media & $\overline{D E}$ & Media & $\overline{D E}$ & Media & $\mathrm{DE}$ & \\
\hline Ansiedad & 25.09 & 8.7 & 17.26 & 12.27 & 17.25 & 11.42 & $F(2,120)=4 \cdot 16^{*}$ \\
\hline Depresión & 7.85 & 3.96 & 4.0 & 4.07 & 3.93 & 3.32 & $F(2,120)=9.78^{\ddagger}$ \\
\hline Auto estima & 18.77 & 3.75 & 19.03 & 4.43 & 18.43 & 3.84 & $F(2,120)=0.82 \mathrm{NS}$ \\
\hline Síntomas & 5.81 & 2.94 & 4.86 & 3.15 & 4.83 & 2.53 & $F(2,120)=0.24 \mathrm{NS}$ \\
\hline $\begin{array}{l}N S=\text { no significativo } \\
D E=\text { desviación estándar }\end{array}$ & $\begin{array}{l}* p \\
\neq p\end{array}$ & & & & & & \\
\hline
\end{tabular}


salud no resultaron significativos y pueden observarse en el cuadro IV. Las variables psicosociales se asociaron significativamente con las de ansiedad $\mathrm{F}(10,54)=$ $1.95, p<0.05$; la depresión $\mathrm{F}(10,54)=2.01, p<0.05$, y la autoestima $\mathrm{F}(10,54)=1.61, p<0.05$.

Las mujeres que presentan mayor carga en la planificación y decisiones de las tareas de la casa notifican niveles importantes de ansiedad y depresión. La mayor carga en la planificación relacionada con las comidas se asocia con una menor autoestima por parte de las mujeres. Cuando la mujer tiene la mayor carga de las tareas del hogar relacionadas con la limpieza y el mantenimiento en general del hogar presenta mayores niveles de depresión. En aquellos casos donde la mujer se encarga de la mayoría de las actividades relacionadas con el cuidado de los hijos presenta mayores indicadores de ansiedad.

\section{Discusión}

La incorporación formal de la mujer al mercado de trabajo ha creado cambios que necesitan estudiarse a pro-

\section{Cuadro III \\ DistribuCión DE LAS RESPONSABILIDADES EN EL HOGAR SEGÚN TIPO DE ROLES. Caracas, Venezuela, 1994-1995}

\begin{tabular}{|c|c|c|c|c|c|c|c|c|}
\hline \multirow{2}{*}{\multicolumn{2}{|c|}{ Salud mental y síntoma }} & \multicolumn{2}{|c|}{ Sin pareja, con hijos $n=23$} & \multicolumn{2}{|c|}{ Con pareja, sin hijos $n=33$} & \multicolumn{2}{|c|}{ Con pareja, con hijos $n=67$} & \multirow{2}{*}{ Anova } \\
\hline & & Media & $\mathrm{DE}$ & Media & DE & Media & $\mathrm{DE}$ & \\
\hline Manejo o planificación de & as en el hogar & 36.78 & 6.40 & 33.69 & 1.01 & 32.91 & 3.87 & $F=(2,120)=8^{\ddagger}$ \\
\hline Ejecución de las tareas en & & 93.65 & 14.4 & 87.24 & 4.35 & 86.71 & 13.31 & $\mathrm{~F}=(2,120)=3.06 *$ \\
\hline $\begin{array}{l}\text { NS }=\text { no significativo } \\
D E=\text { desviación estándar }\end{array}$ & $\begin{array}{l}* p<0.05 \\
\neq p<0.001\end{array}$ & & & & & & & \\
\hline
\end{tabular}

\section{Cuadro IV}

ANÁLISIS DE REGRESIÓN MÚLTIPLE JERÁRQUICA DE LAS VARIABLES SOCIODEMOGRÁFICAS Y LA DISTRIBUCIÓN de LAS Responsabilidades en el hogar con VARIABles de SAlud. Caracas, Venezuela, 1994-1995

$$
N=125
$$

Ansiedad
$B^{*}$
Depresión
Autoestima

$\mathrm{B}^{*}$

Paso 1 variables sociodemográficas

\begin{tabular}{lccr}
$\mathrm{r}^{2}$ & .041 & .020 & .171 \\
\hline Edad & -.164 & .066 & .114 \\
\hline Hijos en edad escolar & .031 & .144 & -.107 \\
\hline Horas semanales de trabajo & .041 & .020 & .068 \\
\hline Cargo & .078 & -.016 & -.026
\end{tabular}

Paso 2 variables psicosociales

\begin{tabular}{lccc}
$\mathrm{r}^{2}$ & $.224^{\ddagger}$ & .251 & $.230^{\ddagger}$ \\
\hline Planificación y decisiones en las tareas de la casa & $.329^{\ddagger}$ & $.458^{\S}$ & -.180 \\
\hline Planificación relacionada con las comidas & -.080 & -.156 & $-.367^{\S}$ \\
\hline Realización de tareas de limpieza & .149 & $.447^{\ddagger}$ & -.179 \\
\hline Cuidado de los niños & $.287^{\ddagger}$ & .049 & -.168 \\
\hline O tras tareas (cuidar las plantas, mascotas) & -.068 & -.230 & .347 \\
\hline Mantenimiento del hogar & -.145 & -.114 & .149
\end{tabular}

* En cada paso se reportan los coeficientes de regresión estandarizados para cada variable

NS $=$ no significativo $\quad \neq p<0.05$

$\mathrm{DE}=$ desviación estándar $\quad \S p<0.01$ 
fundidad, ya que a pesar de que se ha flexibilizado la división social del trabajo, según la cual correspondía a las mujeres la responsabilidad del cuidado del hogar y a los hombres el desempeño de actividades propiamente económicas y, en general, públicas, todavía se supone que la responsabilidad de los quehaceres domésticos sigue siendo fundamentalmente de las mujeres, independientemente de su participación o no en el mercado laboral. ${ }^{16}$ Debido a esta realidad se hace imprescindible analizar su impacto y consecuencias en la mujer, tanto en los aspectos negativos como en las fortalezas de esta nueva condición.

En este estudio, las mujeres que notificaron sobrecarga en la planificación de las actividades de la casa refirieron presencia de ansiedad y depresión; la sobrecarga reportada en la planificación de las comidas se relaciona con disminución de la autoestima. La percepción de sobrecarga en la realización de las tareas de limpieza y el mantenimiento del hogar se asoció con depresión. Aquellas mujeres que tuvieron sobrecarga en las actividades relacionadas con el cuidado de los hijos presentaron indicadores de ansiedad. Pareciera que el percibir sobrecarga en la planificación y realización de las tareas del hogar y el cuidado de los hijos está relacionado con una peor salud mental en este grupo de mujeres.

$\mathrm{Al}$ respecto, Biernat y Wortman ${ }^{17}$ en un estudio con parejas del mismo estatus profesional encontraron que las mujeres contribuyen en mayor grado que sus esposos tanto al cuidado de los hijos como con el resto de las actividades de la casa, lo que genera tensión en el rol familiar y de pareja. Lee-Blair y Johnson ${ }^{18}$ señalan que la participación de la pareja es mayor cuando la mujer trabaja fuera del hogar; sin embargo, la mujer tiene siempre una carga mayor de actividades en el hogar que su pareja, específicamente en aquellas actividades relacionadas con la planificación de dichas tareas. Las mujeres trabajadoras perciben beneficios tanto materiales como psicológicos que influyen y amplían su desarrollo no sólo profesional sino también personal; pero cuando la mujer asume otras responsabilidades como el cuidado de los hijos y las tareas de la casa, esto representa demandas que a su vez influyen en su salud y bienestar general. ${ }^{10}$

En este estudio no fue posible evaluar el grado real de participación de los esposos referido por ellos mismos; sin embargo, según el informe de las mujeres, la participación de sus parejas en las actividades fue menor que la de ellas, tomando en cuenta que la gran mayoría de las participantes cuenta con la ayuda del servicio doméstico. Este factor de participación de los esposos o parejas se relaciona directamente con la percepción que tiene la mujer de sobrecarga tanto en la planificación como en la ejecución de las tareas del hogar y el cuidado de los hijos, ampliamente reseñado en la literatura especializada. $3,7,8,17$

Como se mencionó anteriormente, la sobrecarga percibida en las responsabilidades de la casa está también relacionada con el nivel de participación de la pareja y la equidad que la mujer perciba en esta distribución. ${ }^{7}$ Estos autores, Piña y Bengtson, ${ }^{7}$ señalan que en mujeres casadas, trabajadoras de tiempo completo, el grado de satisfacción y bienestar está influenciado por la distribución equitativa de las responsabilidades en el hogar y el apoyo percibido por parte de su pareja. En este sentido, Yogev y Brett ${ }^{11}$ encontraron que la mujer trabajadora tenía altos niveles de satisfacción marital cuando sus esposos participaban en las actividades del hogar. Barnett y Shan, ${ }^{19}$ detectaron que el grado de sobrecarga y estrés en cuanto a las responsabilidades en el hogar, notificado tanto por las mujeres trabajadoras como por sus parejas, se asoció con determinadas tareas de la casa como preparar las comidas diariamente, asear la cocina luego de éstas, limpiar la casa y realizar compras en el mercado.

En una investigación, realizada por Feldman y colaboradores, ${ }^{*}$ con mujeres trabajadoras de tres niveles ocupacionales diferentes: profesionales, secretarias, personal medio y obreras, encontraron que a mayor sobrecarga en las tareas del hogar, interferencia en los roles entre sí y percepción de un menor control, se notificó mayor número de síntomas asociados tanto con problemas de salud física como mental.

Es así como la distribución de las responsabilidades en el hogar constituye un aspecto fundamental que acompaña e influye en la percepción de la cualidad de los roles, bien moderando los efectos o bien comportándose como generador de mayores demandas que repercute directamente en la salud de la mujer. Este aspecto debe ser tomado en cuenta en futuras investigaciones para profundizar en sus efectos y cómo es su comportamiento en otros grupos poblacionales.

Con relación a las medidas de salud utilizadas tanto en el reporte físico como en los indicadores de salud mental (autoestima, depresión y ansiedad), todas se obtuvieron mediante el autoinforme, una medida ampliamente utilizada y reseñada en la literatura. ${ }^{\ddagger 20-22}$ Es de hacer notar la inclusión de una medida positi-

\footnotetext{
* Feldman L, Chacón G, Blanco G, Carrasquel Y. Proyecto de investigación mujer-trabajo y salud. Decanato de Investigaciones. Caracas: Universidad Simón Bolívar, 1995. Publicación interna.

‡ Feldman L. Temor a hablar en público: Evaluación del componente subjetivo. Trabajo de ascenso para optar a la categoría de Profesor Agregado. Caracas:Universidad Simón Bolívar, 1989. Publicación interna.
} 
va, la autoestima, en la evaluación de la salud mental, aspecto sugerido por Meneses ${ }^{23}$ y Feldman, ${ }^{*}$ en sus respectivos estudios locales. Por otra parte, el índice de morbilidad utilizado para evaluar la salud física probablemente no resultó ser suficientemente discriminativo ya que no se relacionó de modo significativo con ninguna de las variables sociodemográficas y psicosociales; por lo anterior, se sugiere que la evaluación de la salud física debe ser complementada con medidas de tipo fisiológico específicas para contar así con medidas objetivas como aditamento del autoinforme; este aspecto se debe tomar en cuenta para nuevos estudios.

En cuanto a la salud física llama la atención que $32 \%$ de las participantes consideraron que su salud estaba peor que hace cinco años, esta cifra sugiere que la percepción del deterioro al pasar de los años es una realidad para la cuarta parte del grupo, a pesar de tratarse de mujeres jóvenes (el promedio de edad es de 40 años); lo que podría estar relacionado con la sobrecarga en las responsabilidades en el hogar, tomada como una variable contribuyente.

En cuanto a salud mental, este grupo de mujeres notificó buena salud mental y muy pocos síntomas, lo que a su vez se relaciona con lo señalado en relación con la salud física, resultando ser mujeres saludables en su gran mayoría. Sin embargo, la literatura reseña que las mujeres muestran puntajes más altos que los hombres en los aspectos negativos del funcionamiento mental como son depresión, ansiedad y estrés psicológico. ${ }^{9,24}$ En una revisión realizada por Doyal, ${ }^{25}$ el autor señala que las mujeres que trabajan tiempo completo fuera del hogar y que tienen hijos en edad escolar tienen mayor riesgo de presentar depresión.

Estos hallazgos pudieran estar relacionados con la falta de poder y de control que tiene la mujer con relación al hombre dentro de la familia; de acuerdo con la ideología de los roles sociales con relación al género, la mujer es la responsable de las tareas de la casa. Como consecuencia de esto, el empleo constituye un elemento adicional de demanda y, por ende, de sobrecarga al agregarse a las otras actividades que tiene que realizar la mujer regularmente. ${ }^{25}$ Pareciera que aún cuando las demandas existen éstas no llegan a deteriorar de una manera importante la salud mental, al menos en este grupo de mujeres docentes.

Por otra parte, aunque estas mujeres son profesionales, así como la mayoría de sus parejas, sería

\footnotetext{
* Feldman, L. Factores psicosociales asociados a la salud de la mujer con roles múltiples. Trabajo de ascenso para optar a la categoría de Profesor Asociado. Caracas: Universidad Simón Bolívar, 1995.
}

importante tomar en cuenta a futuro el balance de los ingresos familiares. Los hombres suelen ganar más dinero que las mujeres por trabajos de igual nivel. Como consecuencia el balance en los ingresos familiares podría hacer que se desviara la carga de actividades extralaborales (domésticas) hacia la persona con menos ingresos relativos. Es importante tomar en consideración este aspecto para futuros estudios.

Es necesario resaltar que estos resultados están enmarcados en un grupo o sector específico del total de la población de mujeres trabajadoras; debido a que son docentes universitarias con un nivel educativo y socioeconómico determinado hace que los resultados se particularicen respecto a su generalización. Se recomienda realizar futuras investigaciones en otros grupos de mujeres trabajadoras (obreras, secretarias, gerentes, etc.) para así categorizar tanto las semejanzas como las diferencias que se encuentran en las variables estudiadas.

\section{Referencias}

1. Matthews K, Rodin J.W omen's work and family roles: Sources of stress and sources of strength. En: Fisher S, Reason J, ed. Handbook of life stress, cognition and health. N ueva York: John W illey \& Sons, 1989.

2. Di Benedetto B, Jher TC. Gender and adult role: Role commitment of women and men in job-family trade-off context.J Counsel Psychol 1990; 37(1):41-48.

3.W iersma U,Van D en Berg P.W ork-home role conflict, family climate, and domestic responsabilities among men and women in dual-earner families. J A ppl Soc Psychol 1991;21(15):1207-1217.

4.A Imeida, D, Maggs J, Galambos N .W ives' employment hours and spousel participation in family work. J Fam Psychol 1993; 7(2); 233-244.

5. Mederer H. Division of labor in two-earner homes: Task accomplishment versus household management as critical variables in perceptions about family work. J Marriage Fam 1993;55:133-145.

6. Barañano M. Mujer, trabajo, salud. Madrid: Editorial Trotta, 1992.

7. Piña, D, Bengtson V.The division of household labor and wives' happiness: Ideology, employment, and perceptions of support. J Marriage Fam 1993:55:901-912.

8. Baruch G, Barnett R. Role quality, multiple role involvement, and psychological well-being in midlife women. J Pers Soc Psychol 1986;51(3):578585.

9. Rosenfield S. The cost of sharing: W ives' employment and husbands mental health. J Health Soc Behav 1992;33:213-225.

10. Major B. Gender, entitlement, and the distribution of family labor.J Soc Issues 1993;49(3):141-159.

11. Yogev S, Brett J. Perceptions of division of housework and child-care and marital satisfaction.J Marriage Fam 1985; 47:609-618.

12. $N$ ewell $\mathrm{S}$.The superwoman syndrome: $G$ ender differences in attitudes towards equal oportunities at work and towards domestic responsabilities at home.W ork Employ Soc 1993;7(2):275-289.

13. D erogatis LR, Lipman RS, C ovi L, Rickles K. N eurotic symptom dimensions. Arch Gen Psychiatry 1971;24:454-464.

14. Johnson M.The reliability of the derived factor structure for two scales measuring different aspects of self-esteem to be tested by confirmatory 
factor analysis. D octoral Dissertation. D epartament of Psychology. Estocolmo: Stockholm University, 1993.

15. Canino E, Groeger C, Robles J.Adaptación del cuestionario de excitabilidad individual; confiabilidad, análisis de ítemes y estructura factorial. Argos 1994;19:43-66.

16. Ministerio de Asuntos Sociales. Mujeres latinoamericanas en cifras. Bogotá: Flacso, 1995.

17. Biernat $M, W$ ortman $C$. Sharing of home responsabilities between professional employed women and their husbands. J Pers Soc Psychol 1991; 60(6):844-860.

18. Lee-Blair S, Johnson M.W ive's perceptions of the fairness of the division of household labor:The intersection of housework and ideology. J Marriage Fam 1992;54:570-581.

19. Barnett R, Shan YCh. Gender, high and low-schedule-control. Housework task and psychological distress. J Fam Issues 1997;18(4):403-428.
20. Segovia J, Bartlett R, Ewards A.T The association between self-assessed health status and individual health practices.C an J Public Health 1989;80: 32-37.

21. Moun T. Self-assessed health among N orwegian adults. Soc Sci Med 1992;35(7):935-947.

22. Smith A, Shelley J, D ennerstein L. Self-rated health: Biological continuum or social discontinuity? Soc Sci Med 1994;1:77-83.

23. Meneses R. D esempeño de roles múltiples y salud de la mujer.Tesis de Maestría en Psicología. C aracas: Universidad Simón Bolívar, 1994.

24. Baum A, Grunberg N. Gender, stress and health. Health Psychol 1991;10:80-85.

25. Doyal L. Hazards and home.W omen's Studies Int Forum 1990;3(5): 501-517. 